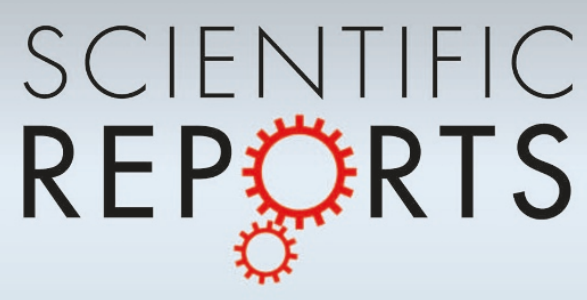

OPEN

SUBJECT AREAS:

ATOMISTIC MODELS

MECHANICAL ENGINEERING

APPLIED PHYSICS

Received

3 April 2014

Accepted

28 August 2014

Published

17 September 2014

Correspondence and requests for materials should be addressed to

B.L.H. (mebhuang@

ust.hk)

\section{Computational Study of In-Plane Phonon Transport in Si Thin Films}

\author{
Xinjiang Wang' \& Baoling Huang ${ }^{1,2}$
}

${ }^{1}$ Department of Mechanical and Aerospace Engineering, The Hong Kong University of Science and Technology, Clear Water Bay,
Kowloon, Hong Kong, ${ }^{2}$ The Hong Kong University of Science and Technology Shenzhen Research Institute, Shenzhen, 518057, China.

We have systematically investigated the in-plane thermal transport in Si thin films using an approach based on the first-principles calculations and lattice dynamics. The effects of phonon mode depletion induced by the phonon confinement and the corresponding variation in interphonon scattering, which may be important for the thermal conductivities of ultra-thin films but are often neglected in precedent studies, are considered in this study. The in-plane thermal conductivities of Si thin films with different thicknesses have been predicted over a temperature range from $80 \mathrm{~K}$ to $800 \mathrm{~K}$ and excellent agreements with experimental results are found. The validities of adopting the bulk phonon properties and gray approximation of surface specularity in thin film studies have been clarified. It is found that in ultra-thin films, while the phonon depletion will reduce the thermal conductivity of Si thin films, its effect is largely offset by the reduction in the interphonon scattering rate. The contributions of different phonon modes to the thermal transport and isotope effects in Si films with different thicknesses under various temperatures are also analyzed.

ingle crystalline silicon thin films have been widely used in microfabricated sensors, actuators, and transistors ${ }^{1,2}$. The efforts in reducing the power consumption and miniaturizing microelectronic devices entail even thinner silicon thin films. Ultra-thin Si films with a thickness less than $12 \mathrm{~nm}$ have been fabricated and applied to Silicon-On-Insulator (SOI) transistors ${ }^{3}$ to lower drive voltages. However, experiments show that attenuating the film thickness below submicron can significantly suppress the in-plane thermal transport, resulting in a thermal conductivity several factors or even an order of magnitude lower than that of the corresponding bulk material ${ }^{4-6}$. The reduction of thermal conductivity in $\mathrm{Si}$ thin films can deteriorate the heat dissipation process in electronic devices such as SOI-based microprocessors ${ }^{7}$, light-emitting diode (LED) and differential scanning nanocalorimeters ${ }^{8}$, where good lateral heat conduction is crucial for efficient thermal management, fast thermal response and good sensitivity. On the other hand, this size effect may serve as a promising solution to enhance the figure of merit when applied to thermoelectric devices ${ }^{9}$. Understanding and predicting spectral thermal transport in thin films is essential to further the advance of the nanoelectronics and thermoelectrics. However, currently the measurements of in-plane thermal conductivity of Si thin films haven't forged deeper into the sub-20 nm regime ${ }^{5}$, calling for theoretical efforts to investigate the phonon transport in such ultra-thin films.

With the attenuation of film thickness, phonon scattering at the film boundaries becomes relatively more important in suppressing phonon mean free path (MFP). This has been widely accepted as the main reason for the reduction of thermal conductivity in nanostructured materials $s^{10,11}$. Early-stage investigations have presented intuitive comparisons between the phonon MFP and the structure size $e^{4}$. The phonon scattering by boundaries essentially depends on both the surface roughness and the incident phonon wavelength ${ }^{12,13}$. This dependence is often characterized by the specularity parameter $p$, which indicates the fraction of phonon energy being specularly reflected by the boundary. For ultra-thin thin films, the confinement of phonons can significantly modify phonon properties such as the dispersion relation ${ }^{14}$. Due to the requirement of forming standing waves ${ }^{15}$, the wavelengths of existing phonon modes must comply with the constraint by the film boundaries ${ }^{13}$. Accordingly, phonon properties such as the density of states (DOS), group velocity and heat capacity, are modified, as has been confirmed by previous molecular dynamics (MD) studies ${ }^{16,17}$. Moreover, the change in the phonon dispersions also affects the phonon-phonon interactions, e.g., fewer phonon modes will result in smaller interphonon scattering rates.

Analytical phonon conductivity models based on Boltzmann Transport Equation (BTE), typically in CallawayHolland form ${ }^{18,19}$, have been developed to understand the variation of in-plane thermal conductivity with respect to film thickness through fitting with experimental results ${ }^{6,10}$. These models often adopt bulk phonon properties and include the effects of boundary scattering in the relaxation time term. This might be inappropriate for ultrathin films in which phonon dispersions and scatterings may be different from those in bulk materials. These 
models also rely on multiple fitting parameters and assume isotropic phonon properties, which might obscure the underlying phonon physics $^{16}$. Meanwhile, the specular parameter $p$ for the boundary scattering is often simply assumed to be a constant for all the phonons ${ }^{20,21}$. However, this simplification needs further verification. Thin film studies based on MD simulations ${ }^{16,22}$ have also been conducted to provide atomic-level information about thermal transport. Classical MD results essentially depend on the quality of interatomic potentials. Considering the fact that few classical potentials including the widely-used Stillinger-Weber ${ }^{23}$ and Tersoff $^{24}$ potentials can accurately reproduce the measured thermal conductivity of bulk $\mathrm{Si}$, it is difficult to directly compare $\mathrm{MD}$ results of $\mathrm{Si}$ thin films with experimental data. Meanwhile, MD simulation is strictly valid only near or above the Debye temperature while the Debye temperature of silicon $\left(631 \mathrm{~K}^{25}\right)$ is much higher than the room temperature.

In contrast, lattice dynamics (LD) calculation targets directly on the transport behavior of individual phonon modes. It is usually combined with BTE to obtain lattice thermal conductivity from kinetics theory ${ }^{26}$. Different from those analytical phonon conductivity models, LD usually adopts full phonon dispersions and considers the mode-wise phonon transport properties, making it more accurate and convenient for spectral analysis, which is of importance for nanoengineering thin films for targeted applications. Lattice dynamics calculation has been successfully used to investigate the relationship between in-plane thermal conductivity and film thickness ${ }^{16,27}$. Turney et al. ${ }^{16}$ have implied the importance of considering mode depletion in modeling the thermal transport in thin films. However, few studies have integrated all the aforementioned phonon confinement effects, especially the change in interphonon scatterings, into the prediction of thermal conductivity of thin films. Besides, LD calculation requires the input of phonon dispersion relation and the relaxation time of each phonon mode, which, especially the latter, are often lacking in accurate determination ${ }^{20,28}$. In recent years, first-principles calculations have been successfully used to accurately predict the phonon scattering rates and thermal conductivities in simple bulk crystals such as $\mathrm{Si}$ and $\mathrm{PbTe}^{29,30}$. It is very attractive to use the results from first-principles calculations instead of fitted parameters from experiments as the inputs for LD calculations, which may provide more reliable insights into the thermal transport in thin films.

In this study, we have systematically investigated the in-plane thermal transport in Si thin films over a wide thickness range from $80 \mathrm{~K}$ to $800 \mathrm{~K}$ using the LD method and first-principles calculations. The phonon confinement effects, including the phonon dispersion modification and the change in interphonon scattering, have been integrated into the prediction of thin film thermal conductivity. The relative contributions of these two mechanisms to the thermal transport in ultra-thin films are discussed. The isotope effects in thin films are also explored. The validity of using a constant specularity parameter and bulk phonon properties in thin film studies is further clarified. Thereafter, the effects of the existence of boundary on the spectral phonon transport are discussed.

\section{Results}

A. Phonon properties and isotope effects. The phonon dispersion relations of bulk Si were calculated based on the harmonic force constants obtained by the first-principles calculations, as shown in Fig. 1(a) together with precedent experimental results ${ }^{31}$. The excellent agreement verifies the accuracy of the harmonic properties of Si from this approach. When the film is attenuated to only a few unit cells thick, the phonon confinement effect limits available phonon modes in the thin film, leading to different harmonic and anharmonic phonon properties from those in bulk Si. Figure 1(a) also shows the DOS variation with respect to film thickness due to this phonon confinement effect. The phonon confinement effects on DOS are shown to vanish when the film

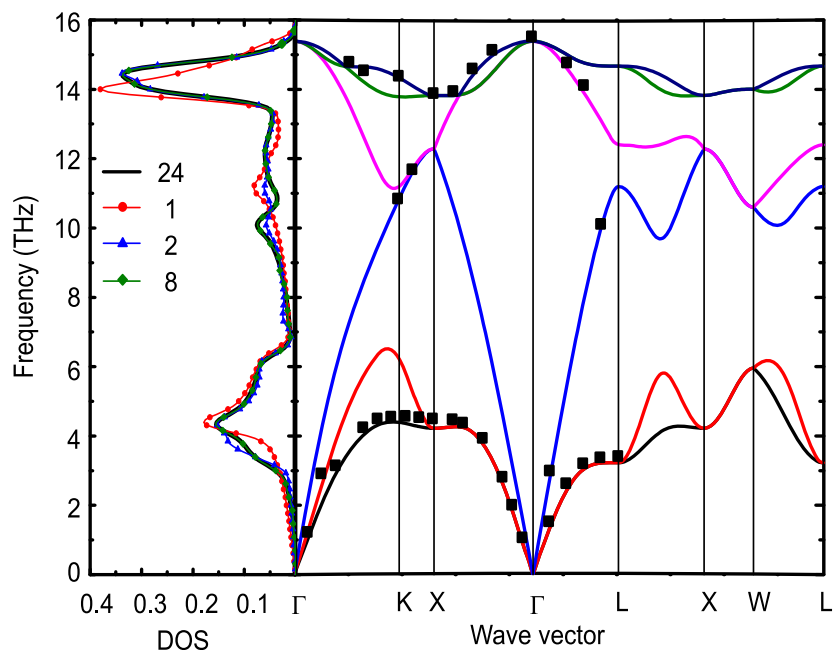

(a)

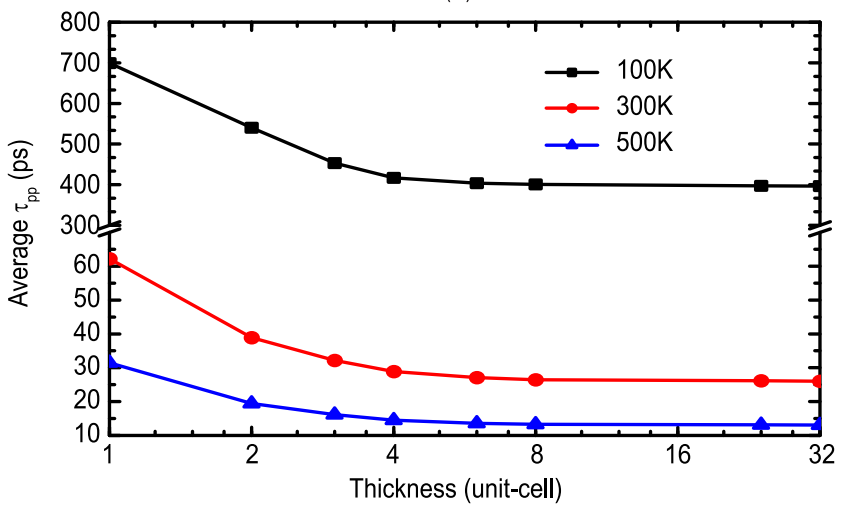

(b)

Figure $1 \mid$ (a) Phonon dispersion relation of bulk silicon along some highsymmetry directions together with the DOS plot for thin films of different thickness (measured in number of unit-cells). The black square dots in the plot of dispersion relation are extracted from the experimental results acquired by G. Nilsson and G. Nelin ${ }^{31}$. (b) Average phonon-phonon scattering relaxation time with respect to the film thickness at different temperatures.

thickness increases to 24 unit cells $(\sim 13 \mathrm{~nm})$. Similar DOS deviation in very thin films has also been observed from classical MD simulations ${ }^{16,21}$. This phonon confinement effect limits available phonon modes in thin film and will also affect the interphonon scattering rate. Figure 1(b) shows the influence of phonon confinement on the average phonon relaxation time limited by the phonon-phonon scattering $\overline{\tau_{p-p}}\left(\overline{\tau_{p-p}}=\frac{\sum_{\xi} C_{v}(\xi) v_{g, x}^{2}(\xi) \tau_{p-p}(\xi)}{\sum_{\xi} C_{v}(\xi) v_{g, x}^{2}(\xi)}\right)$ in thin films of various thicknesses at different temperatures. $\overline{\tau_{p-p}}$ gradually decreases with the increasing film thickness and becomes almost a constant when the thickness is larger than around 24 unit cells $(\sim 13 \mathrm{~nm})$.

The calculated lattice thermal conductivities of bulk silicon at different temperatures are shown in Fig. 2, together with experimental results. The predicted bulk thermal conductivities of ${ }^{28} \mathrm{Si}$ coincide well with the experimental results for ${ }^{28} \mathrm{Si}$ in the temperature range from $80 \mathrm{~K}$ to $300 \mathrm{~K}$ and the predicted bulk thermal conductivities of ${ }^{\text {nat }} \mathrm{Si}$ are also in good agreement $(<15 \%$ error) with the corresponding experimental data from $80 \mathrm{~K}$ to $800 \mathrm{~K}$, highlighting the accuracy of the current approach. At high temperatures $(>800 \mathrm{~K})$, current first-principles calculation tends to overestimate the thermal conductivity because of the ignored higher-order pho- 


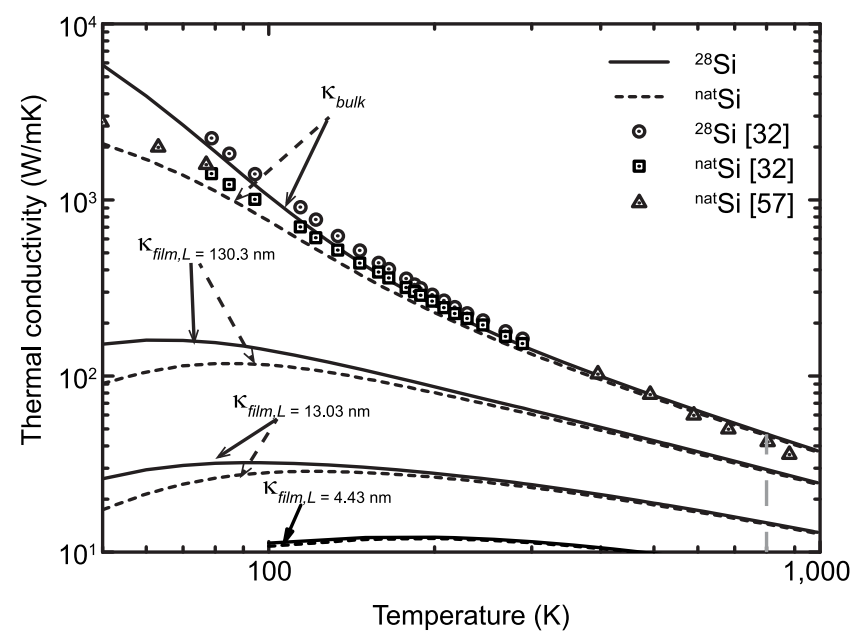

Figure $2 \mid$ Calculated thermal conductivities of bulk $\mathrm{Si}$ and in-plane thermal conductivities of $S i$ thin films with a thickness of $130.3 \mathrm{~nm}$, $13.03 \mathrm{~nm}$ and $4.43 \mathrm{~nm}$ at different temperatures. The solid lines represent results for isotope-enriched samples and the dashed lines represent the results for the samples with natural isotopic abundance. The experimental results $^{32,56}$ for both isotope-enriched ${ }^{28} \mathrm{Si}$ samples and naturally occurring samples are also shown.

non scattering processes, which may play a significant role at elevated temperatures ${ }^{31}$. The calculated thermal conductivity of ${ }^{28} \mathrm{Si}$ at $300 \mathrm{~K}$ is $141 \mathrm{~W} / \mathrm{m}-\mathrm{K}$, slightly lower than the experimental result $155 \mathrm{~W} / \mathrm{m}$ $\mathrm{K}^{32}$, but still close to the result $(\sim 145 \mathrm{~W} / \mathrm{m}-\mathrm{K})$ obtained by Esfarjani using a similar method ${ }^{33}$. This slight underestimation is probably due to the single-mode relaxation time approximation adopted in this work, which may slightly underestimate thermal conductivity, as pointed out by J. Garg et $\mathrm{al}^{34}$. A more accurate method to solve BTE is to use the iterative method, as shown by Chernatynskiy et $\mathrm{al}^{35}$. However, the discrepancy is small for $\mathrm{Si}^{36}$ and is acceptable for thin-film thermal conductivity calculations without fitting with experiments.

Also shown in Fig. 2 are the thermal conductivities of Si thin films with a thickness of $130.3,13.03$, and $4.34 \mathrm{~nm}$. Here the specularity parameter $p$ is set to 0 . Apparently, the results for thin films are much lower than the bulk values over the entire temperature range. Generally a smaller thickness leads to a lower thermal conductivity but a weaker temperature dependence, because of the relatively stronger boundary scattering. When temperature drops from $300 \mathrm{~K}$ to $100 \mathrm{~K}$, the thermal conductivity of bulk ${ }^{28} \mathrm{Si}$ increases almost one order of magnitude while the corresponding improvements for the $130.3-\mathrm{nm}{ }^{28} \mathrm{Si}$ film and $13.03-\mathrm{nm}^{28} \mathrm{Si}$ film are around $170 \%$ and $50 \%$, respectively; however, the thermal conductivity of the 4.43-nm film only decreases slightly. The temperature at which the film thermal conductivity reaches its maximum also increases with the decrease in thickness, probably due to the competition among the boundary scattering, interphonon scattering and specific heat variation. For example, the thermal conductivity of the $13.03 \mathrm{~nm}$ thick ${ }^{28} \mathrm{Si}$ film reaches the maximum at $80 \mathrm{~K}$ while that for the $130.3 \mathrm{~nm}$ thick ${ }^{28} \mathrm{Si}$ film is around $60 \mathrm{~K}$.

The isotope effects are also examined for both bulk silicon and thin films. The thermal conductivity of bulk ${ }^{\text {nat }} \mathrm{Si}$ with natural isotope abundance $\left(92.2 \%{ }^{28} \mathrm{Si}, 4.7 \%{ }^{29} \mathrm{Si}\right.$ and $\left.3.1 \%{ }^{30} \mathrm{Si}\right)$ is predicted as $133 \mathrm{~W} / \mathrm{m}-\mathrm{K}$ at $300 \mathrm{~K}, 6 \%$ lower than that of pure ${ }^{28} \mathrm{Si}$ samples and matching well with the experimental value (7\% in Ref. 32). Figure 2 shows that above $200 \mathrm{~K}$ the curves for bulk pure ${ }^{28} \mathrm{Si}$ and ${ }^{\text {nat }} \mathrm{Si}$ almost overlap, indicating that the interphonon scattering overwhelms the isotope scattering at high temperatures. Below $200 \mathrm{~K}$, the gap between the bulk thermal conductivities of ${ }^{28} \mathrm{Si}$ and ${ }^{\text {nat }} \mathrm{Si}$ increases with the decreasing temperature, in agreement with the results from

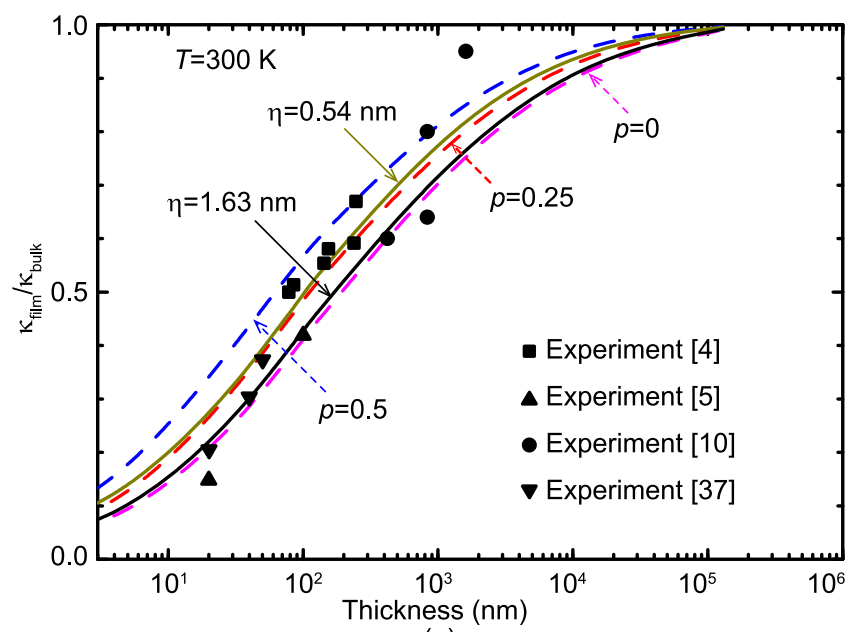

(a)

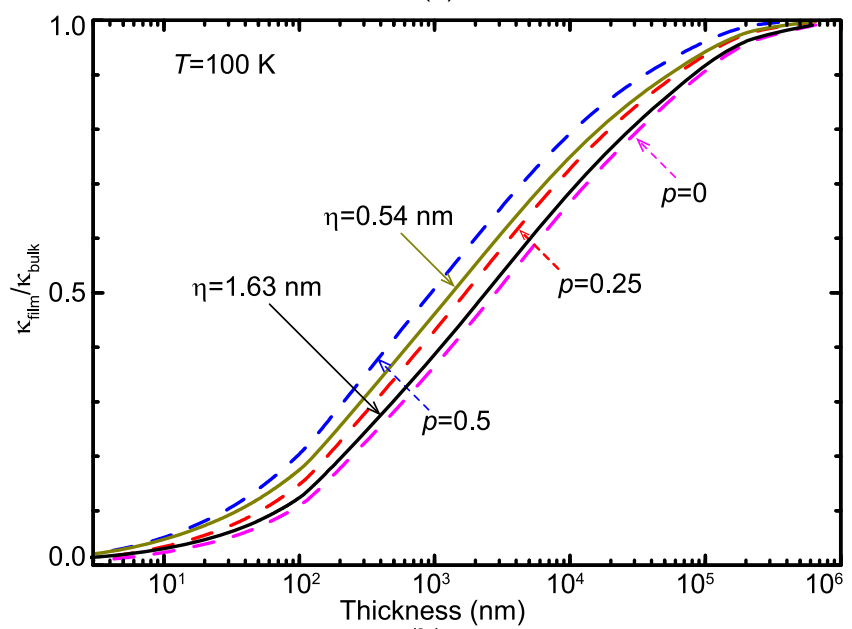

(b)

Figure 3 Variation of thermal conductivity of Si thin films with respect to the film thickness at (a) $300 \mathrm{~K}$ and (b) $100 \mathrm{~K}$. Different values of the specularity parameter $p$, which are either pre-specified or determined by the surface roughness $\eta$, are used in the calculation.

Lindsay et $\mathrm{al}^{36}$. Although similar phenomena can be found in thin films and the isotopes start to play a role below $200 \mathrm{~K}$, the isotope effects are not significant and become even weaker with the decrease in thickness, implying that the isotope effect cannot match the dominant boundary scattering. For the $4.34 \mathrm{~nm}$ film, the isotope effects are almost negligible even at low temperatures. The existence of isotopes will also slightly shift the temperature at which the thermal conductivity reaches the maximum to the right side, e.g., the corresponding temperature for the $13.03 \mathrm{~nm}$ film is shifted from $80 \mathrm{~K}$ to around $100 \mathrm{~K}$. Overall the isotope effects are not important for thin films at elevated temperatures and they are ignored in the following discussions unless specified otherwise.

B. Boundary scattering and phonon depletion in thin films. As implied in Eq. (8) in the method section, the specularity parameter $p$, which depends on both phonon wavelength and surface roughness, directly affects thermal conductivities of thin films. However, a constant $p$ value is often used for convenience in thermal conductivity calculations $s^{20,21}$. To check the validity of such a gray approximation, by setting $p$ as some predetermined constants or a parameter dependent on surface quality and phonon characteristics (Eq. (9)), thermal conductivities of $\mathrm{Si}$ thin films with various thicknesses at $300 \mathrm{~K}$ are calculated and shown together with experimental results ${ }^{4,5,10,37}$ in Fig. 3(a). Apparently, as observed in experiments, the thermal conductivity of thin film decreases with the 
attenuation of film thickness. Figure 3(a) also illustrates that at $300 \mathrm{~K}$ the effects of boundary scattering may be significant for Si thin films of a thickness below $10 \mu \mathrm{m}$ (corresponding to $90 \%$ of the bulk value). Most experimental results fall into the region between $p=0$ and $p=$ 0.5 curves, implying rather diffusive scattering conditions on the film surface. As the film thickness decreases, the thermal conductivity of thin films is more sensitive to $p$, because the boundary scattering becomes more important for thermal transport. When $p$ is determined by the phonon wavelengths and pre-assigned average surface roughness $\eta$, it is found that the results agree quite well with those obtained by assuming a constant $p$ for all the phonon modes. Even though $\eta=0.54 \mathrm{~nm}$ approaches a constant $p=0.25$, a totally diffuse boundary condition $(p=0)$ is still reasonable for most samples since a roughness of only 1 unit cell $(0.54 \mathrm{~nm})$ is almost the achievable minimum roughness during fabrication, not to mention the possible existence of a native oxide layer (around $1 \mathrm{~nm}$ ) on the surfaces of many Si thin films. A similar conclusion has also been drawn from recent experiments ${ }^{38}$ on the spectral transmission in Si thin films. It should be noted that the current model neglects the thickness of native oxide layer and disordered Si surface layer on the Si surface, which may lead to a thermal conductivity slightly higher than the corresponding experimental results. However, the assumption that the specularity can be treated as a constant does not strictly hold at low temperatures, as shown for $T=100 \mathrm{~K}$ in Fig. 3(b). The $\eta=0.54 \mathrm{~nm}$ curve, which almost overlaps with the $p=0.25$ curve over the entire thickness range at $300 \mathrm{~K}$, deviates from the $p=0.25$ curve and approaches the $p=0.5$ curve when the thickness is below $100 \mathrm{~nm}$. This is because the heat-carrying phonons have a longer average wave length at lower temperatures, enabling them less likely to be scattered diffusively at thin film boundary. But for films with a large $\eta$, e.g., $\eta=1.63 \mathrm{~nm}, p=0$ is still a good approximation at $100 \mathrm{~K}$, indicating a completely diffusive scattering even at low temperatures for surfaces with a large roughness because the dominant phonon wavelength is still not long compared with $\eta$ at this temperature. This agrees well with the recent experimental result ${ }^{37}$. As expected, Fig. 3 shows that the boundary scattering effects will be relatively stronger at low temperatures.

For ultra-thin films with thickness within the sub-10 nm regime, the boundary scattering, phonon depletion effect and revision of interphonon scattering rate will all contribute to the change of thermal conductivity. However, their individual contribution is yet to be clarified. In Fig. 4, the thermal conductivities of Si thin films with respect to film thickness at $300 \mathrm{~K}$ are plotted considering various confinement effects. When the bulk phonon properties $\left(C_{v}, v_{g}\right.$ and $\left.\tau_{p-p}\right)$ are used and only the boundary scattering is considered, a conventional treatment of boundary effects (denoted as " $\mathrm{B}$ "), the thermal conductivity decreases with the reduction of film thickness due to stronger boundary scattering. It is obvious that when the phonon depletion effect due to phonon confinement is also considered (denoted as "B + D"), thermal conductivity can be further reduced because less phonons contribute to thermal transport. The thinner the film is, the fewer phonon modes there exist in the thin film, rendering a larger gap between the " $B$ " and " $B+D$ " curves. However, fewer phonons also result in larger interphonon scattering relaxation times, which can offset the effect of phonon depletion. Considering all these three confinement effects (denoted as "B + $\mathrm{D}+\mathrm{R}$ "), the calculated thermal conductivities are much higher than the values for the case " $\mathrm{B}+\mathrm{D}$ ", but they are only slightly lower than those for the case " $\mathrm{B}$ " where only the boundary scattering is considered. The small discrepancy between the " $\mathrm{B}+\mathrm{D}+\mathrm{R}$ " line and the " $\mathrm{B}$ " line indicates that the phonon depletion effect is largely cancelled by the relaxation time revision. Therefore, the conventionally used "crude" model still works well for ultra-thin films accidentally. Recently Cuffe et $\mathrm{al}^{39}$ reported the measurement of the phonon lifetimes of an ultra-thin film as thin as $8 \mathrm{~nm}$. In their analytical fitting

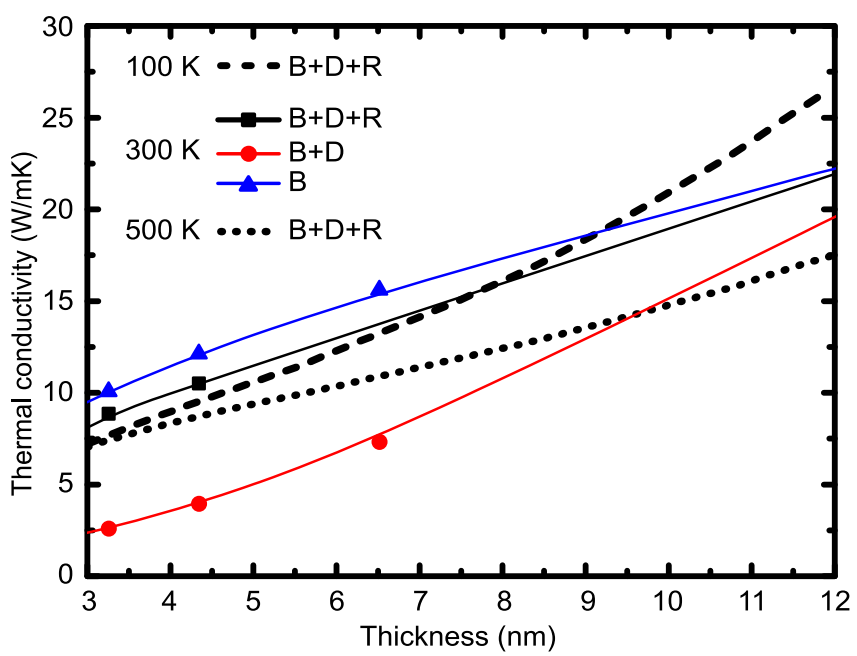

Figure $4 \mid$ Thermal conductivity in ultra-thin Si films with $1 \mathrm{~nm}$ surface roughness at $300 \mathrm{~K}$ constrained by different conditions. " $\mathrm{B}$ " represents phonon boundary scattering, " $D$ " represents the phonon mode depletion effect and " $R$ " denotes the revision of relaxation time of phonons due to phonon mode depletion in thin films. " $D+R$ " indicates the confinement on phonon, including both the wave length constraint and phonon relaxation time revision.

part, a simple boundary scattering was added into the phonon-phonon scattering term without considering the phonon depletion effect, which should be important in the sub-10 nm region. However, the fitting results turned to be satisfactory, implying that the phonon mode depletion effect and relaxation time revision effect may counterbalance with each other in such a thin film, which coincides with the current research.

In the frame of " $\mathrm{B}+\mathrm{D}+\mathrm{R}$ ", when temperature is also taken into consideration, some unique behaviors can be observed for ultra-thin films. First of all, for ultra-thin films, the thermal conductivity is almost temperature-independent. For a thin film with thickness of $3 \mathrm{~nm}$, the thermal conductivity changes less than 15\% when the temperature rises from $100 \mathrm{~K}$ to $500 \mathrm{~K}$, a phenomenon similar to previous $\mathrm{MD}$ results ${ }^{40}$. This indicates that the boundary scattering dominates the thermal transport in ultra-thin films in this temperature range. Also shown in Fig. 4 is a crossover between the thermal conductivity variations at $100 \mathrm{~K}$ and $300 \mathrm{~K}$. The crossover means that even when silicon has a higher thermal conductivity at $100 \mathrm{~K}$ than at $300 \mathrm{~K}$, if the film thickness shrinks to a few nanometers, the situation may be reversed. It is related to the phonon excitation and its interplay with the boundary scattering. For relatively thick films in which the interphonon scattering plays an important role, fewer phonon modes are excited and there are fewer interphonon scattering processes at $100 \mathrm{~K}$ than at $300 \mathrm{~K}$, resulting in larger phonon relaxation times or longer MFPs. However, for ultra-thin films, the phonon MFP is essentially determined by the boundary scattering and fewer phonons will be excited to contribute to thermal transport at lower temperatures, resulting in a decrease in the thermal conductivity.

Figure 5(a) shows the thermal conductivity and the contributions from each phonon branch of bulk silicon and a 130.3-nm-thick thin film (denoted with dashed lines and solid lines, respectively) with respect to the temperature. For bulk $\mathrm{Si}$, the thermal conductivity decreases inversely proportional to the temperature due to the increasing Umklapp scattering strength. The optical phonons (including LO, TO1 and TO2) contribute little to the bulk thermal conductivity and their contributions are insensitive to temperature variation above $200 \mathrm{~K}$. At lower temperatures (<200 K), optical phonons hold less contributions while acoustic phonons contributes 

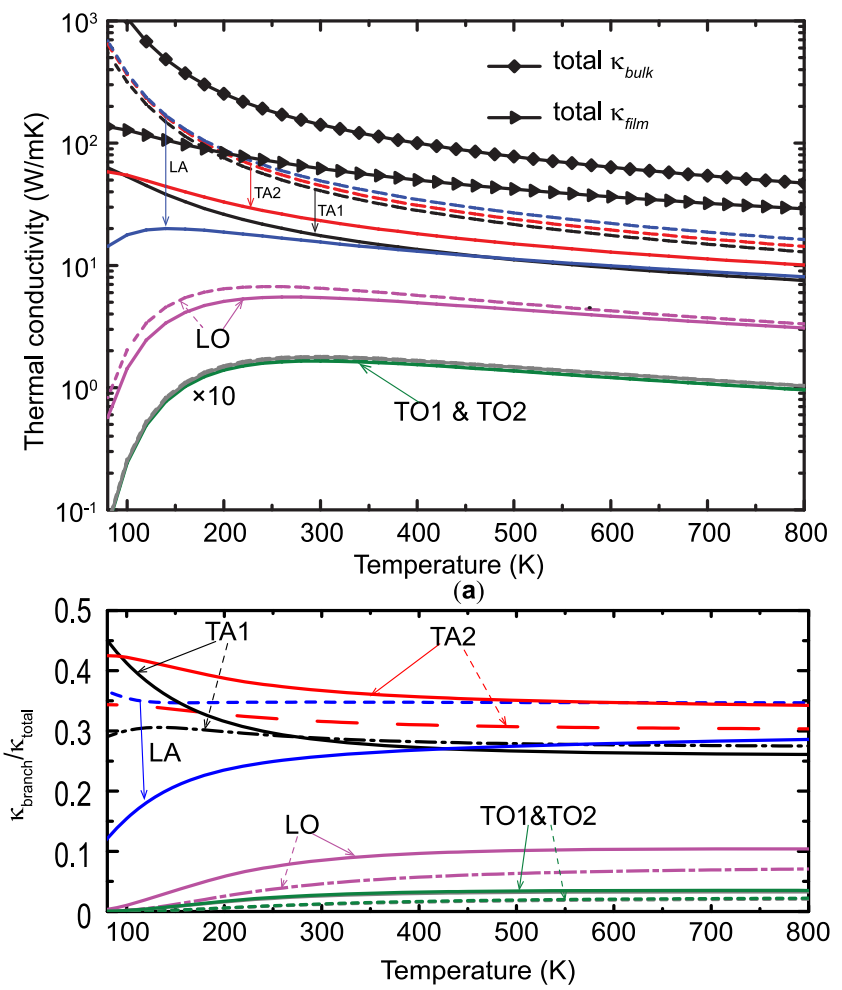

(b)

Figure $5 \mid$ (a) Thermal conductivity contributions from different phonon branches at different temperatures. (b) Relative thermal conductivity contributions from different branches at different temperatures. The dashed lines and solid lines denote bulk $\mathrm{Si}$ and the Si thin film, respectively. The thickness of the thin film is $130.3 \mathrm{~nm}$. The values of TO1 and TO2 in (a) have been multiplied by 10 . Scattering processes at the boundaries of the thin films are considered as purely diffusive, namely, $p=0$.

even more, because less optical phonons are excited below $200 \mathrm{~K}$. The thermal conductivity of bulk Si is dominated by the acoustic phonon branches. Among them, LA modes contribute the most (around $35 \%$ at $300 \mathrm{~K}$ ) while the contributions from TA1 $(28 \%$ at $300 \mathrm{~K})$ and TA2 $(32 \%$ at $300 \mathrm{~K})$ are slightly smaller. For the thin film, similar phenomenon is observed, except that the absolute thermal conductivity contributions from acoustic phonons have been suppressed substantially while the contributions from optical phonons are almost intact because of their intrinsic short MFPs limited by the interphonon scattering. The contribution from LA modes also becomes less than those from TA modes below $400 \mathrm{~K}$, indicating LA modes are more sensitive to the boundary scattering due to their relatively longer MFPs. Moreover, at low temperatures, unlike in bulk $\mathrm{Si}$, the absolute LA contribution to the thermal conductivity of thin films does not always increase with the decreasing temperature. Instead, the absolute contribution of LA modes decreases when the temperature drops below $130 \mathrm{~K}$. Meanwhile, the contributions from TA modes still increase with the decrease in temperature. That is because the cut-off frequency of the LA branch is much higher than those of TA branches (shown in Fig. 1(a)) and there are much more high-frequency modes in the LA branch. At low temperatures, the MFPs of these high-frequency acoustic modes are mainly limited by the boundary scattering but their populations decrease significantly with the decreasing temperature, resulting in smaller contributions to thermal transport. Figure 5(b) shows the relative contribution of each phonon branch in bulk $\mathrm{Si}$ and the thin film with respect to temperature. For both bulk Si and the thin film, the relative contributions from optical phonons increase with the

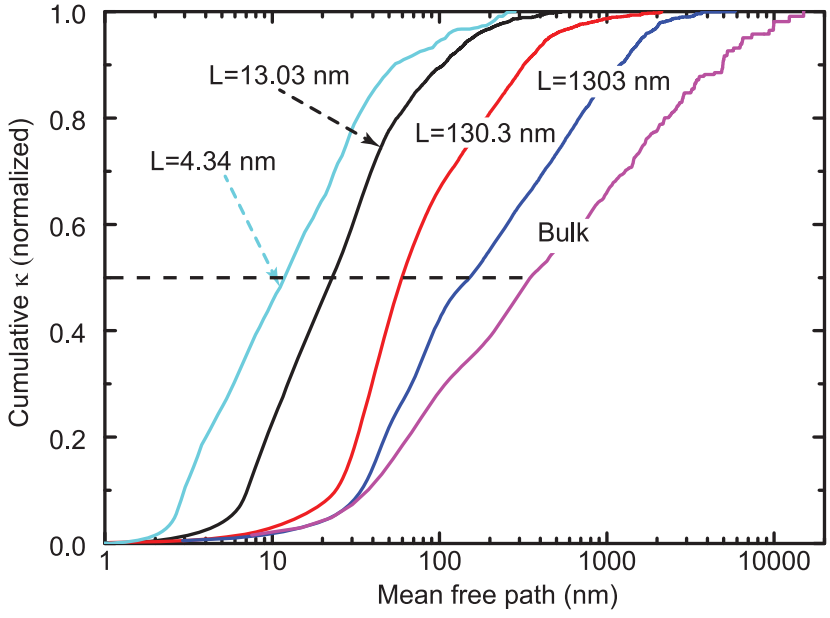

Figure 6 | Normalized thermal conductivity accumulation for bulk Si and Si thin films of different thicknesses with respect to phonon mean free path at $300 \mathrm{~K}$.

increasing temperature and start to saturate (around 20\% and 10\% in total for the thin film and bulk $\mathrm{Si}$, respectively) when the temperature is above $400 \mathrm{~K}$. For bulk $\mathrm{Si}$, the relative contributions of the three acoustic branches vary little with temperature; however, for the thin film, the relative contribution from the LA branch decreases significantly below $400 \mathrm{~K}$ and the TA branches dominate at low temperatures.

\section{Discussion}

To understand the effects of the presence of boundary scattering on the phonon MFP and thermal conductivity, by assuming a diffusive boundary condition $(p=0)$, the normalized thermal conductivity accumulations with respect to the phonon MFP for bulk Si and Si thin films of different thicknesses at $300 \mathrm{~K}$ have been calculated and are shown in Fig. 6. For bulk Si, although the average phonon MFP in the entire Brillouin zone is calculated to be $47 \mathrm{~nm}$, phonons with a MFP longer than $100 \mathrm{~nm}$ contribute around $70 \%$ to the thermal conductivity while those with a MFP longer than $1 \mu \mathrm{m}$ still contribute about $30 \%$ to the thermal conductivity. This agrees with the conclusions from previous studies ${ }^{41}$ that a gray approximation may lead to large uncertainties in the analysis. The MFP corresponding to $50 \%$ thermal conductivity accumulation for bulk $\mathrm{Si}$ is $350 \mathrm{~nm}$, in good agreement with the effective MFP $(\sim 300 \mathrm{~nm})$ of dominant phonons derived from experimental conclusion ${ }^{4}$. For $\mathrm{Si}$ thin films, as thickness decreases, the relative contributions from phonons with a short MFP increase although the absolute contributions change little while those from long-MFP phonons decrease, leading to the shrinkage in the span of MFP of dominant phonons. Similar results have been reported in a previous work from Monte-Carlo simulations $^{42}$. As shown in Fig. 6, the MFP value corresponding to $50 \%$ contribution to thermal conductivity also decreases and becomes more and more comparable to the film thicknesses when the thickness is reduced, indicating stronger boundary scatterings in lowthickness films. Meanwhile, it should be noted that for thin films phonons with a MFP much longer than the film thickness still contribute significantly to the thermal conductivity. This indicates that phonons with a large incident angle contribute more to the in-plane thermal transport.

Figure 7 shows the MFP distribution with respect to the frequency. For bulk $\mathrm{Si}$, the distribution of MFP is significantly uneven and generally phonon MFP decreases rapidly with the increasing frequency. The distribution of MFPs of phonons in bulk Si demonstrates the notable contributions of low-frequency acoustic phonons to thermal transport. The sudden drops in MFP near 4 

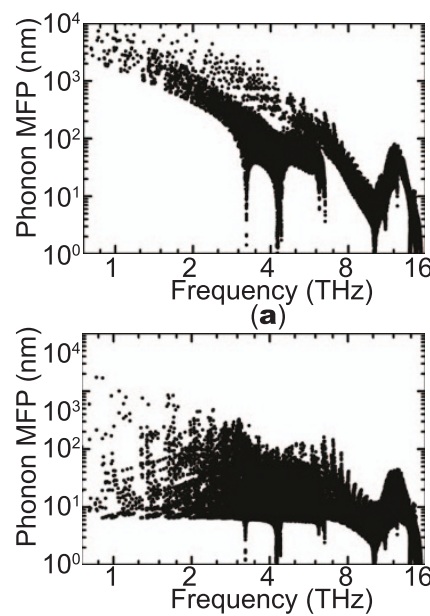

(c)

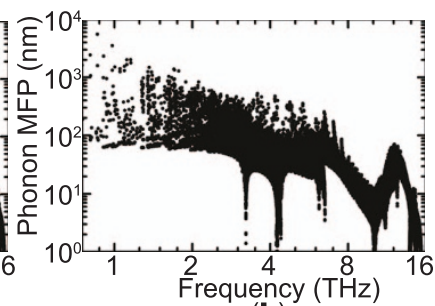

(b)

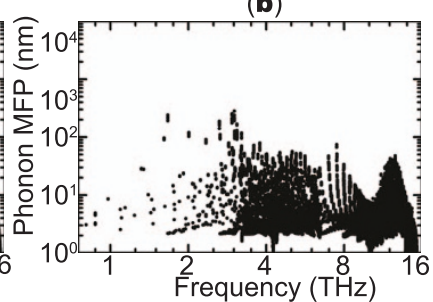

(d)

Figure 7 | Phonon mean free path distribution with respect to frequency at $300 \mathrm{~K}$ for (a) bulk Si and thin films with a thickness of (b) $130.3 \mathrm{~nm}$, (c) $13.03 \mathrm{~nm}$ and (d) $4.34 \mathrm{~nm}$.

and $9 \mathrm{THz}$, corresponding to the cut-off frequencies of the TA and LA branches, are due to the low group velocities at the Brillouin zone boundary. Unsurprisingly, optical phonons generally have short MFPs $(<\sim 10 \mathrm{~nm})$. In contrast, in thin films, the MFP distributions become more even. The MFPs of low-frequency acoustic phonons are significantly reduced by the boundary scattering while the MFPs of high-frequency acoustic phonons and optical phonons are much less influenced. However, some low-frequency phonons still remain their original values because their cross-plane group velocities are relatively small, resulting in a smaller scattering rate on the boundary.

The frequency-dependent contributions to the thermal conductivity for bulk Si and Si thin films with different thicknesses at $300 \mathrm{~K}$ are presented in Fig. 8(a). The inset in Fig. 8(a) shows the cumulative thermal conductivity contribution with respect to frequency. Phonons with frequency below $6 \mathrm{THz}$ dominate the thermal transport in bulk Si and these thin films while optical phonons (with frequency from $11 \mathrm{THz}$ to $15.4 \mathrm{THz}$ ) contribute only $5 \%$ to the thermal conductivity of bulk $\mathrm{Si}$, in line with previous reports ${ }^{33}$. The contributions from low-frequency phonons will be significantly suppressed by the boundary scattering. Both the peak contribution magnitude and position for bulk Si are shifted in thin films while the contributions of high frequency phonons are much less sensitive to the boundaries unless the film thickness is close to their MFP $(\sim 10 \mathrm{~nm})$, similar to the less affected participation ratio of optical phonons in porous structures ${ }^{43}$. Figure 8(b) shows the thermal conductivity contribution with respect to phonon frequency in a 13.03$\mathrm{nm}$ Si thin film at different temperatures. The normalized cumulative contributions are shown in the inset. With the decreasing temperature, the contributions from low-frequency phonons (below $6 \mathrm{THz}$ ) increase while those from the high-frequency regime $(>11 \mathrm{THz})$ decrease, probably due to the reduction in the interphonon scattering rate and the population of high-frequency phonons. The influence of isotopes on the thermal conductivity contributions is also explored and shown in Fig. 8. Generally, as shown in Fig. 8(a), for both bulk Si and thin films at $300 \mathrm{~K}$, the isotope effects are small and they mainly suppress the contributions from acoustic phonons with a frequency between 3 and $5 \mathrm{THz}$. When film thickness decreases, the boundary scattering becomes stronger and the isotope effects become relatively weaker. Figure $8(\mathrm{~b})$ also shows that at $100 \mathrm{~K}$, the isotope effect becomes important and it mainly reduces the contributions from acoustic phonons with a frequency between 3 and $5 \mathrm{THz}$. However, at $300 \mathrm{~K}$ and $500 \mathrm{~K}$, the influence of isotopes is negligible, consistent with previous discussions.

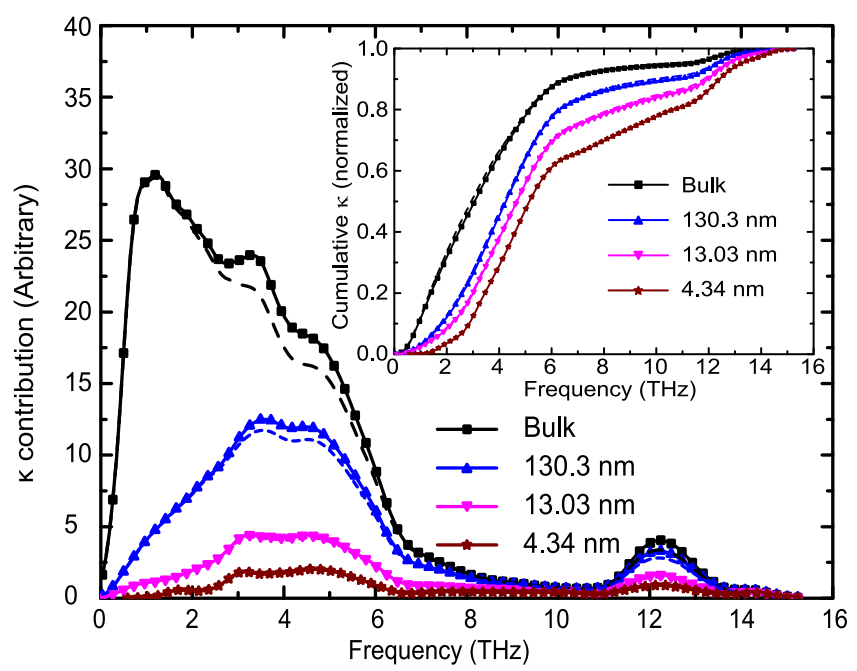

(a)

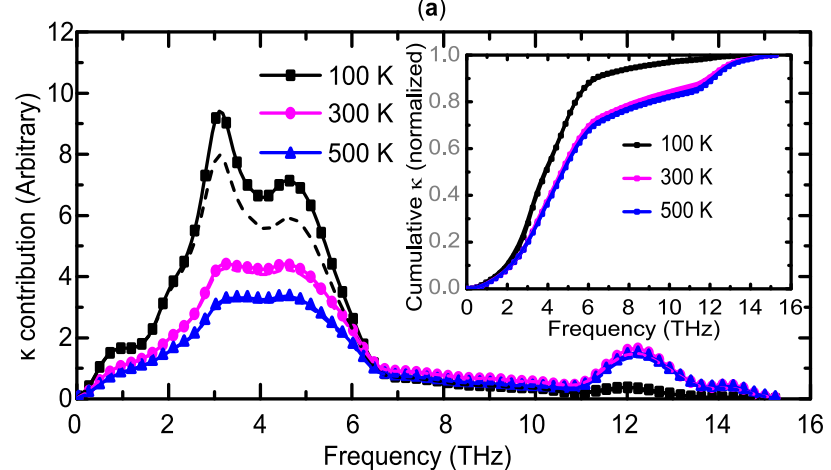

(b)

Figure $8 \mid$ (a) Thermal conductivity contribution with respect to phonon frequency for bulk Si and Si thin films of a thickness of 4.34, 13.03 and $130.3 \mathrm{~nm}$ at $300 \mathrm{~K}$. (b) Thermal conductivity contribution of frequency for the $13.03 \mathrm{~nm}$-thick Si films at different temperatures. The solid lines and dashed lines represent the results without and with considering the isotope effects, respectively. The insets show the normalized thermal conductivity accumulations with respect to phonon frequency.

In summary, we have systematically investigated the dependence of thermal conductivity of $\mathrm{Si}$ thin films on various parameters, including thickness, temperature, isotope effect, and surface roughness. The approach adopted is based on phonon BTE with inputs from the first-principles calculations and lattice dynamics, making it possible to directly compare the predictions with experimental results. The good agreement between the predictions and experimental results indicates the validity of this approach. The phonon confinement effects are also considered in this approach. The investigation of the thermal transport in bulk Si and thin films shows that isotope effects are not important above $200 \mathrm{~K}$. For ultra-thin films with a thickness below $\sim 13 \mathrm{~nm}$, phonon depletion induced by the phonon confinement modifies the phonon spectrum but its effect on thermal conductivity is largely offset by the relaxation time variation. The widely-used gray approximation for the specularity factor is only valid at elevated temperatures or for samples with a large surface roughness. The spectrum analysis shows the dominant phonons for thermal transport in thin films, providing information for tailoring thermal conductivity through nanoengineering.

\section{Methods}

Phonon thermal conductivity from lattice dynamics. Lattice thermal conductivity can be calculated by solving the phonon Boltzmann Transport Equation (BTE) with inputs from first principles calculations. Despite the higher accuracy of the iterative solution $^{35}$, the single-mode relaxation time approximation is sufficient in determining thermal conductivity of silicon because of the strong non-conserving 
phonon scattering in the material ${ }^{36,44}$ and is therefore adopted here for its easier implementation and its explicit relationship with phonon mean free path. According to the kinetic theory about phonon transport and applying Fourier's law, the lattice thermal conductivity $\kappa$ can be predicted by ${ }^{34}$ :

$$
\kappa_{\alpha \beta}=\sum_{\xi} C_{v}(\xi) v_{g, \alpha}(\xi) v_{g, \beta}(\xi) \tau(\xi)
$$

where $\kappa$ is a $2^{\text {nd }}$-order tensor with the subscripts $\alpha$ and $\beta$ denoting its components, $C_{v}$ is the phonon mode heat capacity, $\mathbf{v}_{\mathbf{g}}=\partial \omega / \partial \mathbf{q}$ is the group velocity and $\tau$ is the phonon relaxation time. This summation is over all phonon modes $\xi(\mathbf{q}, j)$ denoted by the wavevector $\mathbf{q}$ and the dispersion branch $j$.

In Eq. (1), $C_{v}, v_{g}$ and $\tau$ are all mode-dependent. The phonon mode heat capacity $C_{v}$ is calculated through

$$
C_{v}=\frac{k_{B}}{V}\left[\frac{\hbar \omega(\xi)}{k_{B} T}\right]^{2} \frac{\exp \left(\hbar \omega(\xi) / k_{B} T\right)}{\left[\exp \left(\hbar \omega(\xi) / k_{B} T\right)-1\right]^{2}}
$$

where $\omega$ is the phonon angular frequency, $k_{B}$ is the Boltzmann constant, $T$ is the temperature and $V$ is the volume of the unit cell respectively. Phonon mode heat capacity and group velocity can be determined from the dispersion relations obtained by solving the eigenvalue problem through diagonalizing the Fourier-transformed harmonic interatomic force constants (dynamical matrix) ${ }^{45}$.

Phonon relaxation time $\tau$ indicates the scattering strength of phonons. In Si crystal, there are generally various scattering mechanisms that limit the phonon transport, including intrinsic phonon-phonon scattering, boundary scattering, and phonondefect scattering (isotope scattering in this study). Matthiessen's rule is often adopted to sum up the effects of independent multiple scattering mechanisms

$$
\frac{1}{\tau_{\text {total }}}=\sum_{i} \frac{1}{\tau_{i}}=\frac{1}{\tau_{p-p}}+\frac{1}{\tau_{p-b}}+\frac{1}{\tau_{p-i}} \ldots
$$

Here $\tau_{p-p}, \tau_{p-b}$ and $\tau_{p-i}$ are the phonon relaxation times limited by the phononphonon scattering, boundary scattering, and phonon-isotope scattering, respectively.

Phonon-phonon scattering. For silicon thermal conductivity calculation, the term of intrinsic phonon-phonon scattering $\tau_{p-p}$ can be predicted by anharmonic LD calculations. Although it is possible to consider the forth-order or even higher order inharmonic effects in LD calculations, which might be important at very high temperatures, three-phonon scattering is the dominant phonon-phonon scattering process below $1000 \mathrm{~K}$. In this work we consider the intrinsic three-phonon scattering process and then the relaxation time $\tau_{p-p}$ is related to the third-order anharmonic interactions ${ }^{29}$

$$
\frac{1}{\tau_{p-p}(\xi)}=\pi \sum_{\xi^{\prime}, \xi^{\prime \prime}}\left|\tilde{V}_{3}\left(-\xi, \xi^{\prime}, \xi^{\prime \prime}\right)\right|^{2} \times\left[\begin{array}{c}
2\left(\bar{n}_{\xi^{\prime}}-\bar{n}_{\xi^{\prime \prime}}\right) \delta\left(\omega(\xi)+\omega\left(\xi^{\prime}\right)-\omega\left(\xi^{\prime \prime}\right)\right) \\
+\left(1+\bar{n}_{\xi^{\prime}}+\bar{n}_{\xi^{\prime \prime}}\right) \delta\left(\omega(\xi)-\omega\left(\xi^{\prime}\right)-\omega\left(\xi^{\prime \prime}\right)\right)
\end{array}\right]
$$

where $\bar{n}$ is the equilibrium Bose-Einstein distribution function of phonons. In this process, the conservation of energy and momentum should be satisfied, i.e., $\omega(\xi) \pm$ $\omega\left(\xi^{\prime}\right)=\omega\left(\xi^{\prime \prime}\right)$ and $\mathbf{q} \pm \mathbf{q}^{\prime}=\mathbf{q}^{\prime \prime}+\mathbf{G}$, where $\mathbf{G}$ is either 0 (for normal process) or a reciprocal lattice vector (for Umklapp process). The interaction strength $\tilde{V}_{3}\left(\xi, \xi^{\prime}, \xi^{\prime \prime}\right)$ among three phonon eigen modes $\xi, \xi^{\prime}$ and $\xi^{\prime \prime}$ can be determined by:

$$
\tilde{V}_{3}\left(\xi, \xi^{\prime}, \xi^{\prime \prime}\right)=\left(\frac{\hbar}{8 N_{0} \omega(\xi) \omega\left(\xi^{\prime}\right) \omega\left(\xi^{\prime \prime}\right)}\right)^{1 / 2} \sum_{b, l^{\prime} b^{\prime}, l^{\prime \prime} b^{\prime \prime}} \sum_{\alpha, \beta, \gamma}\left[\begin{array}{l}
\Psi_{\alpha \beta \gamma}\left(0 b, l^{\prime} b^{\prime}, l^{\prime \prime} b^{\prime \prime}\right) e^{i\left(q^{\prime} l^{\prime}+q^{\prime \prime} l^{\prime \prime}\right)} \\
\times \frac{\mathbf{e}_{\alpha}(b \mid \xi)}{\sqrt{m_{b}}} \frac{\mathbf{e}_{\beta}\left(b^{\prime} \mid \xi^{\prime}\right)}{\sqrt{m_{b^{\prime}}}} \frac{\mathbf{e}_{\gamma}\left(b^{\prime \prime} \mid \xi^{\prime \prime}\right)}{\sqrt{m_{b^{\prime \prime}}}}
\end{array}\right] \text { (5) }
$$

where e is the phonon mode eigenvector and $\Psi_{\alpha \beta \gamma}\left(0 b, l^{\prime} b^{\prime}, l^{\prime \prime} b^{\prime \prime}\right)$ represents the thirdorder force constants in terms of atoms $b, b^{\prime}$ and $b^{\prime \prime}$ in lattice $0, l^{\prime}$ and $l^{\prime \prime}$ of a crystal consisting of $N_{0}$ cells.

Isotope scattering. For isotopes, the mass variation model is often adopted with the mass variance given by

$$
g=\frac{\sum_{i} f_{i} M_{i}^{2}-\left(\sum_{i} f_{i} M_{i}\right)^{2}}{\left(\sum_{i} f_{i} M_{i}\right)^{2}}
$$

where $f_{i}$ and $M_{i}$ are the fraction and mass of the $i^{t h}$ specie of atoms, respectively. According to the model proposed by Tamura ${ }^{46}$, the corresponding relaxation time can be calculated as:

$\tau_{p-i}^{-1}(\xi)=\frac{\pi \omega(\xi)}{2\left(\bar{n}_{\xi}+1\right) N_{0}} \sum_{\xi^{\prime}}\left[\omega\left(\xi^{\prime}\right)\left(\bar{n}_{\xi^{\prime}}+1\right) \delta\left(\omega(\xi)-\omega\left(\xi^{\prime}\right)\right) \sum_{b, \alpha}\left(g(b) \mathbf{e}_{\alpha}^{*}(b \mid \xi) \mathbf{e}_{\alpha}\left(b \mid \xi^{\prime}\right)\right)^{2}\right]$

Two different silicon samples, i.e., pure ${ }^{28} \mathrm{Si}$ and naturally occurring $\mathrm{Si}$ which consists of $92.2 \%{ }^{28} \mathrm{Si}, 4.7 \%{ }^{29} \mathrm{Si}$ and $3.1 \%{ }^{30} \mathrm{Si}$, are considered in this study to illustrate the isotope effects.

Boundary scattering. Based on the accurately calculated bulk phonon properties, the phonon transport process in thin films can be analyzed accordingly. Assume the coordinate $\mathrm{z}$ is along the cross-plane direction, the interactions between phonons and the boundary can be quantified as

$$
\tau_{p-b}=\frac{1+p}{1-p} \frac{L}{2\left|v_{g, z}\right|} .
$$

Here $\frac{L}{2\left|v_{g, z}\right|}$ represents the average time duration for a phonon to reach the boundary, provided evenly distributed possibility of emission location and emission angle. The specularity parameter $p$, ranging from 0 to 1 , is used to account for the possibility of specular reflection on the surface. It can be either specified as a constant value or determined through the relationship ${ }^{47}$

$$
p(\lambda)=\exp \left(-\frac{16 \pi^{3} \eta^{2}}{\lambda^{2}}\right),
$$

where $\lambda$ is the phonon wavelength and $\eta$ is the average surface roughness.

Phonon confinement. When the film thickness is comparable to that of wavelength confinement from the boundaries may affect the thermal transport by changing the wave characteristics of phonon ${ }^{16,48}$. This effect is referred to in the current manuscript as "phonon confinement". It is obliged by the requirement of forming standing waves in the thin film, i.e., phonon wave vector magnitude $q$, which is inversely proportional to the wavelength, should satisfy the constraint

$$
q_{z} \cdot L_{z}=2 \pi \cdot n
$$

Here $L_{z}$ is the thickness of thin film and $n$ is an integer number. Therefore, in the cross-plane direction, only waves that are accommodated by the thickness of the thin film can exists, leading to a change in phonon dispersion relations and phonon DOS.

The confinement effect applies for an infinite potential well or a periodic boundary condition ${ }^{48}$. Whereas for an ultra-thin film, the surface reconstruction ${ }^{49}$ and the existence of native silicon dioxide layer would defy these assumptions. However, these disorders only affect the first few atomic layers near the surface ${ }^{50}$. For Si films of a thickness larger than $3 \mathrm{~nm}$ in this study, the majority of atoms still stay on their lattice sites as in bulk Si. Because of the short-range covalent nature of Si-Si bonds, the interatomic force constants among these inner atoms will not change with the thickness, which has been confirmed in our first-principles calculations. Therefore, the vibration spectra of the main body of the film are close to those of a corresponding film embedded in bulk (see the supplemental materials), but under the constraint of Eq. (10), meaning that the LD calculation is still valid for ultra-thin films. The influence from the surface disorder can be considered in the specularity factor $p$. Then we can obtain the interphonon scattering rate of each mode in thin films according to Eqs. (4) and (5) by only considering the available modes in thin films.

Since the thermal conductivity is obtained by summing up over all the q-points in the Brillouin zone, it can be expected from first sight that the phonon depletion will result in a further decrease of thermal conductivity. However, once the phonon confinement effect is taken into consideration, the assumption that phonon relaxation time remains the original value as in the bulk material is no longer valid. Because of fewer phonons available in the Brillouin zone, some of the original scattering channels which satisfy the momentum conservation relation shown in Eq. (4) don't comply with the standing wave requirement and therefore the interphonon scatterings become weaker. Different from previous studies that adopt bulk interphonon relaxation times in the calculation, in this study the relaxation times of thin film phonon modes are recalculated by only considering the three-phonon scatterings among the available modes in thin films.

Computation details. In this study, a conventional unit cell of silicon was first relaxed within the density-functional theory framework under the Perdew-Burke-Ernzerhof $(\mathrm{PBE})^{51}$ form of the generalized gradient approximation (GGA) for the projector augmented wave method ${ }^{52}$ (PAW) implemented in the Vienna Ab initio simulation package (VASP) ${ }^{53}$. The cut-off energy was set as $500 \mathrm{eV}$. It yielded a lattice constant $a=5.429 \AA$, perfectly agreeing with the experimental value $5.431 \AA^{54}$. Then a $2 \times 2 \times$ 2 supercell was constructed. Thereafter, the so-called direct method ${ }^{55}$ was applied to this supercell to calculate the second and third-order force constants. In this method, atoms were displaced in independent directions. The second-order force constants $\phi_{\alpha \beta}\left(l b, l^{\prime} b^{\prime}\right)=\frac{\partial^{2} E}{\partial u_{\alpha}(l b) \partial u_{\beta}\left(l^{\prime} b^{\prime}\right)}\left(u_{\alpha}(l b)\right.$ is the $\alpha$ coordinate of the $b^{\text {th }}$ atom inside the $l^{\text {th }}$ unit cell) and the third-order force constants $\phi_{\alpha \beta}\left(l b, l^{\prime} b^{\prime}, l^{\prime \prime} b^{\prime \prime}\right)=\frac{\partial^{3} E}{\partial u_{\alpha}(l b) \partial u_{\beta}\left(l^{\prime} b^{\prime}\right) \partial u_{\gamma}\left(l^{\prime \prime} b^{\prime \prime}\right)}$ can then be extracted from the energy and force variation. The static first-principles calculations were conducted with a precision as high as $10^{-8} \mathrm{eV}$ for the total energy difference between two selfconsistency steps and $5 \times 5 \times 5 \mathrm{k}$-points (reciprocal space mesh for electronic selfconsistent field calculation) to obtain the forces on each atom within the perturbed systems. After the extraction of the harmonic and anharmonic force constants, the frequency and relaxation time of each mode were calculated by conducting a Fourier transformation with a dense $\mathrm{q}$ mesh (reciprocal space mesh for phonons) scheme $24 \times 24 \times 24$, which has been tested to be enough to yield converged results in the temperature range from $50 \mathrm{~K}$ to $1000 \mathrm{~K}$. Further increase in the mesh scale has little effect on the predicted bulk thermal conductivity values. 
For thin film calculations only considering phonon depletion effect but no scattering rate revision, we extract the phonon properties, such as phonon frequency and inter phonon scattering relaxation time $\tau_{p-p}$, from bulk phonon modes, with the consideration of the constraint on phonon wavelength [Eq. (10)]. The group velocities of these available modes in the thin film are also recalculated.

However, for ultra-thin films, both the mode depletion and scattering rate revision effects should be considered. The phonon properties in ultra-thin films, including the group velocities and interphonon scattering relaxation times, cannot be extracted from the bulk phonon properties any more, but rather need to be recalculated. During the calculation, a $24 \times 24 \times N$ q mesh scheme is chosen, where $N$ denotes the number of allowable q-points in the cross-plane direction in the thin film. The interphonon scattering relaxation time $\tau_{p-p}$ of each phonon mode in the thin film systems are recalculated according to Eqs. (4) and (5). Different thin film thickness and surface roughness values are specified in order to analyze their effects on the thermal transport in thin films.

1. King, W. P. et al. Atomic force microscope cantilevers for combined thermomechanical data writing and reading. Appl. Phys. Lett. 78, 1300-1302 (2001).

2. Muralt, P. Ferroelectric thin films for micro-sensors and actuators: a review. J. Micromechanics Microengineering 10, 136 (2000).

3. Yoshimi, M., Cauchy, X. \& Maleville, C. Ultra-thin SOI wafer technologies for 22/ $20 \mathrm{~nm}$ CMOS or beyond. in 2010 10th IEEE Int. Conf. Solid-State Integr. Circuit Technol. ICSICT 997-998 (2010).

4. Ju, Y. S. \& Goodson, K. E. Phonon scattering in silicon films with thickness of order $100 \mathrm{~nm}$. Appl. Phys. Lett. 74, 3005-3007 (1999).

5. Liu, W. \& Asheghi, M. Thermal conductivity measurements of ultra-thin single crystal silicon layers. J. Heat Transf. 128, 75-83 (2005).

6. Liu, W. \& Asheghi, M. Phonon-boundary scattering in ultrathin single-crystal silicon layers. Appl. Phys. Lett. 84, 3819-3821 (2004).

7. Pop, E. \& Goodson, K. E. Thermal phenomena in nanoscale transistors. J. Electron. Packag. 128, 102-108 (2006).

8. Olson, E. A., Efremov, M. Y., Zhang, M., Zhang, Z. \& Allen, L. H. The design and operation of a MEMS differential scanning nanocalorimeter for high-speed heat capacity measurements of ultrathin films. J. Microelectromechanical Syst. 12, 355-364 (2003).

9. Venkatasubramanian, R., Siivola, E., Colpitts, T. \& O'Quinn, B. Thin-film thermoelectric devices with high room-temperature figures of merit. Nature 413, 597-602 (2001).

10. Asheghi, M., Leung, Y. K., Wong, S. S. \& Goodson, K. E. Phonon-boundary scattering in thin silicon layers. Appl. Phys. Lett. 71, 1798 (1997).

11. Johnson, J. A. et al. Direct measurement of room-temperature nondiffusive thermal transport over micron distances in a silicon membrane. Phys. Rev. Lett. 110, 025901 (2013).

12. Aksamija, Z. \& Knezevic, I. Anisotropy and boundary scattering in the lattice thermal conductivity of silicon nanomembranes. Phys. Rev. B 82, 045319 (2010).

13. Zou, J. \& Balandin, A. Development of an ab-initio model of the lattice thermal conductivity in semiconductor thin films and nanowires. MRS Online Proc. Libr. 677 (2001).

14. Balandin, A. \& Wang, K. L. Significant decrease of the lattice thermal conductivity due to phonon confinement in a free-standing semiconductor quantum well. Phys. Rev. B 58, 1544-1549 (1998).

15. Chen, F. et al. Quantum confinement, surface roughness, and the conduction band structure of ultrathin silicon membranes. ACS Nano 4, 2466-2474 (2010).

16. Turney, J. E., McGaughey, A. J. H. \& Amon, C. H. In-plane phonon transport in thin films. J. Appl. Phys. 107, 024317 (2010).

17. Heino, P. Dispersion and thermal resistivity in silicon nanofilms by molecular dynamics. Eur. Phys. J. B 60, 171-179 (2007)

18. Callaway, J. Model for lattice thermal conductivity at low temperatures. Phys. Rev. 113, 1046-1051 (1959)

19. Holland, M. G. Analysis of lattice thermal conductivity. Phys. Rev. 132, 2461-2471 (1963).

20. McGaughey, A. J. H., Landry, E. S., Sellan, D. P. \& Amon, C. H. Size-dependent model for thin film and nanowire thermal conductivity. Appl. Phys. Lett. 99, 131904 (2011)

21. Karamitaheri, H., Neophytou, N. \& Kosina, H. Ballistic phonon transport in ultrathin silicon layers: Effects of confinement and orientation. J. Appl. Phys. 113 204305 (2013)

22. Gomes, C. J., Madrid, M., Goicochea, J. V. \& Amon, C. H. In-plane and out-ofplane thermal conductivity of silicon thin films predicted by molecular dynamics. J. Heat Transf. 128, 1114-1121 (2006).

23. Zi, J., Zhang, K. \& Xie, X. Modification of Stillinger-Weber potentials for Si and Ge. Phys. Rev. B 41, 12915-12918 (1990).

24. Tersoff, J. New empirical approach for the structure and energy of covalent systems. Phys. Rev. B 37, 6991-7000 (1988).

25. Desai, P. D. Thermodynamic properties of iron and silicon. J. Phys. Chem. Ref. Data 15, 967-983 (1986)

26. Turney, J. E., Landry, E. S., McGaughey, A. J. H. \& Amon, C. H. Predicting phonon properties and thermal conductivity from anharmonic lattice dynamics calculations and molecular dynamics simulations. Phys. Rev. B 79, 064301 (2009).
27. Reinke, C. M. et al. Thermal conductivity prediction of nanoscale phononic crystal slabs using a hybrid lattice dynamics-continuum mechanics technique. AIP Adv. 1, 041403 (2011)

28. Voneshen, D. J. et al. Suppression of thermal conductivity by rattling modes in thermoelectric sodium cobaltate. Nat. Mater. 12, 1028-1032 (2013).

29. Broido, D. A., Malorny, M., Birner, G., Mingo, N. \& Stewart, D. A. Intrinsic lattice thermal conductivity of semiconductors from first principles. Appl. Phys. Lett. 91, 231922 (2007)

30. Tian, Z. et al. Phonon conduction in PbSe, $\mathrm{PbTe}$, and $\mathrm{PbTe}_{1-\mathrm{x}} \mathrm{Se}_{\mathrm{x}}$ from firstprinciples calculations. Phys. Rev. B 85, 184303 (2012).

31. Nilsson, G. \& Nelin, G. Study of the homology between silicon and germanium by thermal-neutron spectrometry. Phys. Rev. B 6, 3777-3786 (1972).

32. Gusev, A. V., Gibin, A. M., Morozkin, O. N., Gavva, V. A. \& Mitin, A. V. Thermal conductivity of ${ }^{28}$ Si from 80 to $300 \mathrm{~K}$. Inorg. Mater. 38, 1100-1102 (2002).

33. Esfarjani, K., Chen, G. \& Stokes, H. T. Heat transport in silicon from firstprinciples calculations. Phys. Rev. B 84, 085204 (2011)

34. Garg, J. Thermal conductivity from first-principles in bulk, disordered, and nanostructured materials. Ph.D. thesis, Massachusetts Institute of Technology, Cambridge, MA (2011).

35. Chernatynskiy, A. \& Phillpot, S. R. Evaluation of computational techniques for solving the Boltzmann transport equation for lattice thermal conductivity calculations. Phys. Rev. B 82, 134301 (2010).

36. Lindsay, L., Broido, D. A. \& Reinecke, T. L. Ab initio thermal transport in compound semiconductors. Phys. Rev. B 87, 165201 (2013).

37. Ju, Y. S. Phonon heat transport in silicon nanostructures. Appl. Phys. Lett. 87, 153106 (2005).

38. Hertzberg, J. B., Aksit, M., Otelaja, O. O., Stewart, D. A. \& Robinson, R. D. Direct measurements of surface scattering in Si nanosheets using a microscale phonon spectrometer: implications for Casimir-limit predicted by Ziman theory. Nano Lett. 14, 403-415 (2014).

39. Cuffe, J. et al. Lifetimes of confined acoustic phonons in ultrathin silicon membranes. Phys. Rev. Lett. 110, 095503 (2013).

40. Chang, T., Weng, C. \& Huang, M. A nonequilibrium molecular dynamics study of in-plane thermal conductivity of silicon thin films. J. Electron. Mater. 39, 1616-1620 (2010).

41. Wang, Z., Alaniz, J. E., Jang, W., Garay, J. E. \& Dames, C. Thermal conductivity of nanocrystalline silicon: importance of grain size and frequency-dependent mean free paths. Nano Lett. 11, 2206-2213 (2011).

42. Jain, A., Yu, Y.-J. \& McGaughey, A. J. H. Phonon transport in periodic silicon nanoporous films with feature sizes greater than $100 \mathrm{~nm}$. Phys. Rev. B 87, 195301 (2013).

43. Yang, L., Yang, N. \& Li, B. Extreme low thermal conductivity in nanoscale 3D Si phononic crystal with spherical pores. Nano Lett. 14, 1734-1738 (2014).

44. Ward, A. \& Broido, D. A. Intrinsic phonon relaxation times from first-principles studies of the thermal conductivities of Si and Ge. Phys. Rev. B 81, 085205 (2010).

45. Togo, A., Oba, F. \& Tanaka, I. First-principles calculations of the ferroelastic transition between rutile-type and $\mathrm{CaCl}_{2}$-type $\mathrm{SiO}_{2}$ at high pressures. Phys. Rev. B 78, 134106 (2008).

46. Tamura, S. Isotope scattering of dispersive phonons in Ge. Phys. Rev. B 27, 858-866 (1983)

47. Ziman, J. M. Electrons and Phonons: The Theory of Transport Phenomena in Solids. (Oxford, Clarendon Press, 1960).

48. Chen, G. Nanoscale Energy Transport and Conversion: A Parallel Treatment of Electrons, Molecules, Phonons, and Photons. (Oxford Univ. Press, London, 2005)

49. Gibson, J. M., McDonald, M. L. \& Unterwald, F. C. Direct imaging of a novel silicon surface reconstruction. Phys. Rev. Lett. 55, 1765-1767 (1985).

50. Chadi, D. J. Atomic and electronic structures of reconstructed $\mathrm{Si}$ (100) surfaces. Phys. Rev. Lett. 43, 43-47 (1979).

51. Perdew, J. P., Burke, K. \& Ernzerhof, M. Generalized gradient approximation made simple. Phys. Rev. Lett. 77, 3865-3868 (1996).

52. Blöchl, P. E. Projector augmented-wave method. Phys. Rev. B 50, 17953-17979 (1994).

53. Kresse, G. \& Furthmüller, J. Efficient iterative schemes for ab initio total-energy calculations using a plane-wave basis set. Phys. Rev. B 54, 11169-11186 (1996).

54. O'Mara, W. C., Herring, R. B. \& Hunt, L. P. Handbook of Semiconductor Silicon Technology. (Noyes Publications, 1990).

55. Esfarjani, K. \& Stokes, H. T. Method to extract anharmonic force constants from first principles calculations. Phys. Rev. B 77, 144112 (2008).

56. Glassbrenner, C. J. \& Slack, G. A. Thermal conductivity of silicon and germanium from $3^{\circ} \mathrm{K}$ to the melting point. Phys. Rev. 134, A1058-A1069 (1964).

\section{Acknowledgments}

This work was supported by the Hong Kong General Research Fund under Grant Nos. 613211 and 623212

\section{Author contributions}

X.W. and B.H. wrote the manuscript. X.W. prepared all the figures. All authors reviewed the manuscript. 


\section{Additional information}

Supplementary information accompanies this paper at http://www.nature.com/ scientificreports

(i) This work is licensed under a Creative Commons Attribution 4.0 Internationa License. The images or other third party material in this article are included in the article's Creative Commons license, unless indicated otherwise in the credit line; if Competing financial interests: The authors declare no competing financial interests. the material is not included under the Creative Commons license, users will need How to cite this article: Wang, X. \& Huang, B. Spectral In-plane Phonon Transport and to obtain permission from the license holder in order to reproduce the material. To view a copy of this license, visit http://creativecommons.org/licenses/by/4.0/ 
SUBJECT AREAS: ATOMISTIC MODELS MECHANICAL ENGINEERING APPLIED PHYSICS

\section{SCIENTIFIC REPORTS:}

$4: 6399$

DOI: $10.1038 /$ srep06399

Published:

17 September 2014

Updated: 20 January 2015
CORRIGENDUM: Computational Study of In-Plane Phonon Transport in Si Thin Films

Xinjiang Wang \& Baoling Huang

The Acknowledgements section in this Article is incomplete.

“This work was supported by the Hong Kong General Research Fund under Grant Nos. 613211 and 623212." should read

"This work was supported by the Hong Kong General Research Fund (Grant Nos. 613211 and 623212) and National Natural Science Foundation of China (Grant No. 51376154)" 